\title{
Prevalência da cisticercose bovina no Estado de São Paulo (Brasil)*
}

\author{
Bovine cysticercosis prevalence in the State of S. Paulo, Brazil
}

\author{
Mônica L. Ungar*t, Pedro M.L. Germano **
}

\begin{abstract}
UNGAR, M.L. \& GERMANO, P.M.L. Prevalência da cisticercose bovina no Estado de São Paulo (Brasil). Rev. Saúde públ., S. Paulo, 26: 167-72, 1992. Estudou-se a prevalência da cisticercose bovina (Cysticercus bovis) no Estado de São Paulo, no ano de 1986, a partir de fichas de matadouros do Estado sob o controle do Serviço de Inspeção Federal (SIF). Para o estudo da distribuição geográfica, adotou-se a divisão político-administrativa do Estado, formada por 11 Regiôes Administrativas (RAs) e a Região Metropolitana (RM), subdivididas em 42 Regiōes de Governo (RGs), abrangendo 572 municípios. Aos valores de prevalência obtidos aplicou-se o teste " $Z$ " para duas proporções. O total de abate foi igual a 896.654 cabeças, tendo sido diagnosticados 48.957 casos de cisticercose, correspondendo a uma prevalência de $5,5 \%$. Obteve-se resultados de prevalência para 385 municipios, todas as RGs, RAs e a RM. Apresentaram resultados estatisticamente significantes 97 municípios, 14 RGs e 4 RAs.
\end{abstract}

Descritores: Cisticercose, veterinária. Doenças dos bovinos, epidemiologia. Matadouros, normas.

\section{Introdução}

O complexo teníase/cisticercose, determinado pela Taenia saginata, apresenta distribuição cosmopolita, estando amplamente difundido na maioria dos países em que há criação bovina. No entanto, o conhecimento da prevalência da doença, tanto no homem quanto nos animais, é deficiente devido à falta de dados sistemáticos, fidedignos e comparáveis ${ }^{18,21}$.

Os valores da prevalência de cisticercose bovina (Cysticercus bovis) provêm dos serviços de inspeçâo veterinária em matadouros ${ }^{17,19}$. Esta inspeção é realizada em vários países do mundo, porém os métodos diagnósticos "post mortem" utilizados, geralmente possuem diferenças entre si. Desta forma, torna-se difícil a comparação dos resultados obtidos pelos diferentes países,

* Baseado na Dissertação de Mestrado intioulada "Prevalência da cisticercose bovina (Cysticercus bovis Goeze, 1782) do Estado de São Paulo, detectada em matadouros do referido Estado, sob o controle do Serviço de Inspeção Federal, no ano de 1986", apresentada em 1990, na Faculdade de Medicina Veterinária e Zootecnia da Universidade de São Paulo.

** Departamento de Medicina Veterinária Preventiva e Saúde Animal da Faculdade de Medicina Veterinária e Zootecnia da Universidade de São Paulo - São Paulo, SP Brasil.

*** Departamento de Prática de Saúde Pública da Faculdade de Saúde Pública da Universidade de São Paulo - São Paulo, SP - Brasil.

Separatas/Reprints: P.M.L. Germano - Av. Dr. Amaldo, 715 - 01246-904 - São Paulo, SP - Brasil.

Publicação financiada pela FAPESP. Processo Saúde Coletiva 91/4994-0 podendo os mesmos ser discutidos apenas de uma maneira genérica ${ }^{1,19}$.

As limitações sobre as informaçōes da ocorrência da cisticercose bovina são, particularmente, verdadeiras no que se refere à América Latina. Schenone e Letonja ${ }^{20}$, cientes deste problema, realizaram um trabalho de revisão bibliográfica e de pesquisa junto a 25 governos da América Latina sobre a ocorrência dessa zoonose. Porém, no que concerne à espécie bovina, só foi possível apresentar resultados para 7 países: Brasil, Chile, Colômbia, El Salvador, México, Nicarágua e Uruguai.

Particularmente, em relação ao Brasil, a cisticercose bovina, de acordo com a FAO ${ }^{11}$, durante o período de 1977 a 1986 , apresentou freqüência rara e esporádica, sendo que, no ano de 1985, a doença foi também classificada como disseminada por todo território nacional. Contudo, os dados referentes aos anos de 1984 e 1986 não foram atualizados pelas autoridades do país.

Ao longo dos anos, alguns pesquisadores apresentaram relatos sobre a ocorrência da cisticercose bovina no Brasil, sendo que poucos foram aqueles que apresentaram os resultados discriminados por região de procedência dos animais (munícipios e/ou Estados) $2,3,6,10,14,15,16$. Assim, os valores referidos nesses trabalhos situaram a prevalência da cisticercose bovina, para alguns Estados do país, entre 0,7\% e 5,3\%.

Levantamento realizado no periódo de 1971 a 1982, a partir de dados referentes" $a$ matadouros sob controle do Servico de Inspeção Federal (SIF), forneceu o valor médio de prevalência de 
cisticercose bovina, no país, igual a $2,2 \% \%^{24}$.

No Estado de São Paulo, os dados oficiais sobre os casos de cisticercose bovina, no período de 1977 a 1986, só foram apresentados nos anos de $1983(3,90 \%)$ e $1984(4,16 \%)^{5}$. Contudo, no trabalho de Calil ${ }^{7}$, realizado a partir de dados obtidos diretamente na Seção de Estatística do Serviço de Inspeção de Produto Animal (SERPA-SP), encontraram-se descritos os totais de casos de cisticercose bovina nos anos de $1980(3,86 \%), 1981(4,16 \%)$ e $1982(4,18 \%)$.

O matadouro desempenha duas funções básicas no que se refere ao complexo teníase/cisticercose ( $T$. saginata). A primeira é participar da prevenção da teníase humana, através da destinação adequada de carcaças e órgãos bovinos cisticercóticos. A segunda é atuar como fonte de dados estatísticos e nosogeográficos, função esta primordial dentro da vigilância sanitária ${ }^{23}$. $O$ diagnóstico da cisticercose, somado à informação de origem do animal, possibilita definir as áreas de ocorrência da doença, bem como a sua quantificação.

O Estado de São Paulo, bem como os outros Estados do Brasil, possui matadouros sob o controle do SIF, que enviam, mensalmente, ao Ministério da Agricultura, responsável por este serviço, fichas contendo dados sobre o movimento dos estabelecimentos, inclusive aqueles referentes à cisticercose bovina. Dentre estes dados, encontram-se os totais de abate e os totais de casos da doença, segundo a procedência dos animais. Porém, o processamento e divulgação dos mesmos são deficientes, perdendo-se, assim, uma importante informação.

O presente trabalho teve como objetivos determinar a prevalência e a distribuição geográfica da cisticercose bovina no Estado de São Paulo, detectada em nível de matadouros, sob o controle do SIF, bem como identificar as áreas de risco em potencial.

\section{Material e Método}

Fotam utilizadas fichas relativas ao movimento mensal de abate de bovinos de 47 estabelecimentos localizados no Estado de São Paulo, sob o controle do SIF, referentes ao período de primeiro de janeiro a 31 de dezembro de 1986.

Com o intuito de assegurar a confiabilidade dos dados utilizados no levantamento, foram selecionadas, para cada matadouro, 5 fichas referentes a todos os meses disponíveis, relacionadas a seguir:
Ficha 1: "Movimento Mensal de Apreensões de Carcaças de Bovinos";

Ficha 2: "Movimento Mensal de Apreensōes de Vísceras";

Ficha 3: "Mapa Demonstrativo de Localização de Tuberculose e Cisticercose";

Ficha 4: "Mapa Demonstrativo de Doenças por Procedência";

Ficha 5: "Mapa Demonstrativo de Procedência com Peso Morto de Bovinos".

Como base de avaliação da confiabilidade dos dados, foram adotados os resultados referentes ao total de casos de cisticercose, total geral de abate de bovinos e total de abate de animais por município de procedência, sendo que estes dados encontram-se discriminados em 4,3 e 2 fichas, respectivamente. Foram determinados os seguintes critérios para a consolidação de dados: presença de 5 fichas de cada conjunto; valores numéricos iguais, referentes a um mesmo resultado; e discriminaçāo dos resultados referentes a bovinos e búfalos. Os conjuntos de fichas que não preencheram qualquer um destes requisitos foram eliminados do levantamento.

Como resultado deste procedimento, dos 482 conjuntos de fichas originais, foram selecionados 387 conjuntos $(80,3 \%)$. Com relaçāo aos 47 matadouros, foram utilizados, integral ou parcialmente, os dados referentes a $42(89,4 \%)$, enquanto que os dados referentes aos 5 restantes $(10,6 \%)$, foram totalmente desconsiderados.

Dos conjuntos de fichas selecionadas, foram processados os dados referentes aos totais de abate e de casos de cisticercose e procedência dos animais, representada pelos municípios do Estado de São Paulo, constantes na ficha intitulada "Mapa Demonstrativo de Doenças por Procedência".

Para a realização da análise estatística foi utilizado o programa CALC-PROP.

Para a apresentação e avaliação dos resultados de prevalência de cisticercose bovina, foi utilizada a divisão político-administrativa do Estado de São Paulo ${ }^{13}$. Segundo esta divisão, o Estado é constituído pela Região Metropolitana (RM) e 11 Regiōes Administrativas (RAs), formadas por 42 Regiōes de Governo (RGs), abrangendo 572 municípios.

Aos resultados obtidos, aplicou-se o Teste " $\mathrm{Z}$ " monocaudal, para duas proporções, com aproximaçāo normal ${ }^{4}$, para se verificar se o valor de prevalência de uma unidade politico-administrativa era estatisticamente maior quando comparado ao valor de prevalência de seu conjunto. Desta forma, foram submetidas ao teste 
Tabela - Prevaléncia da cisticercose bovina e respectiva significância estatística, segundo a Região Metropolitana, as Regiōes Administrativas, as Regiōes de Governo e os municípios do Estado de São Paulo, existentes $\theta$ considerados no levantamento.

\begin{tabular}{|c|c|c|c|c|c|c|c|c|}
\hline \multirow{3}{*}{$\begin{array}{c}\text { Regiâo } \\
\text { Administrativa" }\end{array}$} & \multicolumn{3}{|c|}{ Regiēo de Governo" } & \multicolumn{5}{|c|}{ Municipio } \\
\hline & \multirow{2}{*}{ Nome } & \multirow{2}{*}{ TABAT } & \multirow{2}{*}{$\begin{array}{c}\text { PREV } \\
\% \\
\end{array}$} & \multicolumn{3}{|c|}{ Total } & \multicolumn{2}{|c|}{ SIG } \\
\hline & & & & $E$ & $\mathrm{C}$ & $\%$ & Ne & $\%$ \\
\hline RM & - & 1354 & 3,2 & 38 & 4 & 10,5 & 0 & 0,0 \\
\hline Subtotal & & 1354 & 3,2 & 38 & 4 & 10,5 & 0 & 0,0 \\
\hline $\begin{array}{c}1 \\
\text { Subtotal }\end{array}$ & Registro & $\begin{array}{l}211 \\
211\end{array}$ & $\begin{array}{l}4,7 \\
4,7\end{array}$ & $\begin{array}{l}12 \\
12\end{array}$ & $\begin{array}{l}2 \\
2\end{array}$ & $\begin{array}{l}16,7 \\
16,7\end{array}$ & $\begin{array}{l}1 \\
1\end{array}$ & $\begin{array}{l}50,0 \\
50,0\end{array}$ \\
\hline $2^{\mathbf{s}}$ & Santos & 38 & 7,9 & 8 & 1 & 12,5 & 0 & 0,0 \\
\hline Subtotal & & 38 & 7,9 & 8 & 1 & 12,5 & 0 & 0,0 \\
\hline Subtotal & $\begin{array}{l}\text { Caraguatatuba } \\
\text { Cruzeiro } \\
\text { Guaratinguetá } \\
\text { São José dos Campos } \\
\text { Taubaté }\end{array}$ & $\begin{array}{r}312 \\
305 \\
754 \\
1951 \\
405 \\
3727\end{array}$ & $\begin{array}{l}7,4^{*} \\
3,0 \\
2,1 \\
2,0 \\
2,2 \\
2,6\end{array}$ & $\begin{array}{c}4 \\
7 \\
7 \\
8 \\
10 \\
36\end{array}$ & $\begin{array}{c}1 \\
3 \\
3 \\
4 \\
2 \\
13\end{array}$ & $\begin{array}{l}25,0 \\
42,9 \\
42,9 \\
50,0 \\
20,0 \\
36,1\end{array}$ & $\begin{array}{l}0 \\
0 \\
1 \\
0 \\
0 \\
1\end{array}$ & $\begin{array}{r}0,0 \\
0,0 \\
33,3 \\
0,0 \\
0,0 \\
7,7\end{array}$ \\
\hline Subtotal & $\begin{array}{l}\text { Avaré } \\
\text { Botucatu } \\
\text { Itapetinga } \\
\text { Itapeva } \\
\text { Sorocaba }\end{array}$ & $\begin{array}{r}4346 \\
1469 \\
435 \\
604 \\
1476 \\
8330\end{array}$ & $\begin{array}{c}2,7 \\
7,4^{*} \\
13,6^{*} \\
7,8^{*} \\
4,6 \\
4,8\end{array}$ & $\begin{array}{l}16 \\
11 \\
10 \\
11 \\
15 \\
63\end{array}$ & $\begin{array}{c}10 \\
8 \\
5 \\
4 \\
4 \\
31\end{array}$ & $\begin{array}{l}62,5 \\
72,7 \\
50,0 \\
36,4 \\
26,7 \\
49,2\end{array}$ & $\begin{array}{l}4 \\
1 \\
1 \\
1 \\
0 \\
7\end{array}$ & $\begin{array}{r}40,0 \\
12,5 \\
20,0 \\
25,0 \\
0,0 \\
22,6\end{array}$ \\
\hline Subtotal & $\begin{array}{l}\text { Bragança Paulista } \\
\text { Campinas } \\
\text { Jundiai } \\
\text { Limeira } \\
\text { Piracicaba } \\
\text { Rio Claro } \\
\text { São João da Boa Vista }\end{array}$ & $\begin{array}{r}7980 \\
4262 \\
467 \\
328 \\
1953 \\
1004 \\
1009 \\
17003\end{array}$ & $\begin{array}{c}3,5 \\
6,7^{\star} \\
6,4 \\
4,0 \\
6,0^{\star} \\
5,9 \\
7,8^{\star} \\
5,1\end{array}$ & $\begin{array}{c}14 \\
18 \\
9 \\
8 \\
10 \\
8 \\
16 \\
83\end{array}$ & $\begin{array}{c}8 \\
10 \\
2 \\
2 \\
7 \\
3 \\
9 \\
41\end{array}$ & $\begin{array}{l}57,1 \\
55,6 \\
22,2 \\
25,0 \\
70,0 \\
37,5 \\
56,2 \\
49,4\end{array}$ & $\begin{array}{l}2 \\
2 \\
0 \\
0 \\
1 \\
0 \\
2 \\
7\end{array}$ & $\begin{array}{r}25,0 \\
20,0 \\
0,0 \\
0,0 \\
14,3 \\
0,0 \\
22,2 \\
17,1\end{array}$ \\
\hline Subtotal & $\begin{array}{l}\text { Araraquara } \\
\text { Barretos } \\
\text { Franca } \\
\text { Ribeirão Preto } \\
\text { São Carlos } \\
\text { São Joaquim da Barra }\end{array}$ & $\begin{array}{r}4385 \\
31692 \\
6440 \\
4896 \\
2306 \\
5165 \\
54884\end{array}$ & $\begin{array}{l}6,4 \\
6,2 \\
6,0 \\
7,8^{*} \\
5,6 \\
6,7 \\
6,3^{*}\end{array}$ & $\begin{array}{c}16 \\
18 \\
17 \\
22 \\
7 \\
6 \\
86\end{array}$ & $\begin{array}{l}11 \\
14 \\
15 \\
15 \\
7 \\
6 \\
68\end{array}$ & $\begin{array}{r}68,8 \\
77,8 \\
88,2 \\
68,2 \\
100,0 \\
100,0 \\
79,1\end{array}$ & $\begin{array}{c}1 \\
4 \\
3 \\
4 \\
1 \\
3 \\
16\end{array}$ & $\begin{array}{r}9,1 \\
28,6 \\
20,0 \\
26,7 \\
14,3 \\
50,0 \\
23,5\end{array}$ \\
\hline Subtotal & $\begin{array}{l}\text { Baurú } \\
\text { Jaú } \\
\text { Lins }\end{array}$ & $\begin{array}{c}32354 \\
619 \\
60960 \\
93933\end{array}$ & $\begin{array}{l}8,1^{*} \\
7,1 \\
7,0 \\
7,4^{*}\end{array}$ & $\begin{array}{l}17 \\
10 \\
10 \\
37\end{array}$ & $\begin{array}{c}17 \\
6 \\
10 \\
33\end{array}$ & $\begin{array}{r}100,0 \\
60,0 \\
100,0 \\
89,2\end{array}$ & $\begin{array}{l}6 \\
1 \\
2 \\
9\end{array}$ & $\begin{array}{l}35,3 \\
16,7 \\
20,0 \\
27,3\end{array}$ \\
\hline Subtotal & $\begin{array}{l}\text { Catanduva } \\
\text { Femandópolis } \\
\text { Jales } \\
\text { Sâo José do Rio Preto } \\
\text { Votuporanga }\end{array}$ & $\begin{array}{c}4687 \\
36516 \\
31879 \\
35199 \\
34429 \\
142710\end{array}$ & $\begin{array}{l}8,8^{*} \\
5,7 \\
5,8 \\
6,0 \\
5,3 \\
5,8^{*}\end{array}$ & $\begin{array}{l}13 \\
11 \\
15 \\
28 \\
14 \\
81\end{array}$ & $\begin{array}{c}6 \\
11 \\
15 \\
25 \\
14 \\
71\end{array}$ & $\begin{array}{r}46,2 \\
100,0 \\
100,0 \\
89,3 \\
100,0 \\
87,6\end{array}$ & $\begin{array}{c}0 \\
3 \\
3 \\
4 \\
4 \\
14\end{array}$ & $\begin{array}{r}0,0 \\
27,3 \\
20,0 \\
16,0 \\
28,6 \\
19,7\end{array}$ \\
\hline $\begin{array}{c}9 \\
\text { Subtotal }\end{array}$ & $\begin{array}{l}\text { Andradina } \\
\text { Araçatuba }\end{array}$ & $\begin{array}{l}143317 \\
126598 \\
269915\end{array}$ & $\begin{array}{l}5,1 \\
5,9 \\
5,5\end{array}$ & $\begin{array}{l}10 \\
26 \\
36\end{array}$ & $\begin{array}{l}10 \\
26 \\
36\end{array}$ & $\begin{array}{l}100,0 \\
100,0 \\
100,0\end{array}$ & $\begin{array}{c}4 \\
9 \\
13\end{array}$ & $\begin{array}{l}40,0 \\
34,6 \\
36,1\end{array}$ \\
\hline
\end{tabular}


Tabela (Continuaçåo)

\begin{tabular}{|c|c|c|c|c|c|c|c|c|}
\hline \multirow{3}{*}{$\begin{array}{c}\text { Regiảo } \\
\text { Administrativa** }\end{array}$} & \multicolumn{3}{|c|}{ Regiảo de Govemo } & \multicolumn{5}{|c|}{ Município } \\
\hline & \multirow{2}{*}{ Nome } & \multirow[t]{2}{*}{ TABAT } & \multirow{2}{*}{$\begin{array}{c}\text { PREV } \\
\%\end{array}$} & \multicolumn{3}{|c|}{ Total } & \multicolumn{2}{|c|}{ SIG } \\
\hline & & & & $E$ & $c$ & $\%$ & $\mathrm{Ne}^{2}$ & $\%$ \\
\hline \multirow[t]{3}{*}{$10^{4}$} & Adamantina & 13376 & $8,8^{*}$ & 11 & 11 & 100,0 & 4 & 36,4 \\
\hline & Dracena & 14625 & $6,0^{*}$ & 10 & 10 & 100,0 & 2 & 20,2 \\
\hline & Presidente Prudente & 246575 & 3,9 & 26 & 26 & 100,0 & 14 & 53,8 \\
\hline Sub-Total & & 274576 & 4,2 & 47 & 47 & 100,0 & 20 & 42,6 \\
\hline \multirow[t]{4}{*}{114} & Assis & 6312 & 4,2 & 11 & 9 & 81,8 & 3 & 33,3 \\
\hline & Marilia & 6670 & 7,9 & 13 & 10 & 76,9 & 1 & 10,0 \\
\hline & Ourinhos & 1557 & 6,3 & 10 & 8 & 80,0 & 2 & 25,0 \\
\hline & Tupâ & 15434 & $9,7^{\star}$ & 11 & 11 & 100,0 & 3 & 27,3 \\
\hline Sub-Total & & 29973 & $8.0^{*}$ & 45 & 38 & 84,4 & 9 & 23.7 \\
\hline Total & & 896654 & 5.5 & 572 & 385 & 67.3 & 97 & 25.2 \\
\hline
\end{tabular}

* Estatisticamente significante

* - 100\% das Regiర̄es de Governo, das Regiōes Administrativas e a Regiảo Metropolitana fol contemplado no levantamento.

TABAT = Total de abate (cabecas)

SIG = Estatisticamente significante

$\mathrm{C}=$ Contemplados no levantamento

14 - Registro

2 - Santos

$3^{a}$ - Săo José dos Campos
PREV = Prevalência

$E=$ Existentes

RM = Região Metropolitana

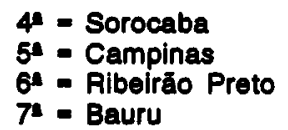

8ะ = Sảo José do Rio Preto

9 - Aracatuba

104 - Presidente Prudente

$11^{2}$ - Marilia as prevalências das seguintes unidades: municípios X RGs; RGs X RAs; e, RAs X Estado.

\section{Resultados — Discussão}

A Tabela apresenta os resultados de prevalência de cisticercose bovina $e$ as respectivas significâncias estatísticas, segundo as RAs e a RM, as RGs e os municípios de São Paulo, existentes e considerados no levantamento.

Com referência aos resultados da prevalência de cisticercose bovina, obtidos no presente levantamento, torna-se difícil estabelecer comparações com resultados de outros trabalhos, devido ao fato de não se ter encontrado publicações com critérios similares, que contivessem a distribuição espacial e temporal da doença no Estado de São Paulo. Desta forma, não é possível avaliar se o valor de prevalência de cisticercose bovina de 5,5\%, obtido para o Estado, em 1986 , bem como os valores específicos das outras unidades político-administrativas, sofreu alguma alteração em relação aos anos anteriores.

Considerando-se as RAs estatisticamente significantes, observa-se, na Tabela, que as mesmas situaram-se entre a sexta e a décima primeira RAs. No conjunto destas 6 RAs, o efetivo abatido correspondeu a $96,6 \%$ do total do Estado (896.654 cabeças). Foram contempladas 23 RGs (54,8\% das 42 existentes), sendo 7 com valores significantes $(50,0 \%$ dos 14 estatisticamen- te significantes). Com relagão aos municípios, dos 572 existentes, $332(58,0 \%)$ pertencem às $R G$ s relacionadas, tendo sido contemplados 293 (76,1\% do total de 385), dos quais, 81 apresentaram significância estatística (83,5\% dos 97 significantes). Deste modo, como se pode observar, todos os resultados, quando avaliados no conjunto da sexta à décima primeira $\mathrm{RA}$, são todos representativos. Deve-se destacar que, em 1986, a somatória do efetivo bovino dessas regiōes, destinado exclusivamente ao corte, representava $80,8 \%$ do total do Estado $^{22}$, o que demonstra a relevante importância desta grande regiāo para a epidemiologia da cisticercose bovina.

As unidades estudadas (RAs, RGs e municípios), que apresentam valores de prevalência estatisticamente significantes, constituem áreas de risco em potencial para a ocorrência da cisticercose bovina. Pode-se considerar como grandes áreas de risco aquelas onde a significância apresentou-se para as 3 unidades estudadas. Enquadraram-se nesta situação 3 RAs, responsáveis por 19,9\% do total geral de abate do Estado, a saber: Ribeirão Preto, Bauru e Marilia, e as RGs Ribeirão Preto, Bauru e Tupã, respectivamente.

Estas áreas também são indicadoras da ocorrência da teníase humana, posto que os animais se infectam através da ingestão de ovos de $T$. saginata, provenientes de fezes humanas. Portanto, os resultados obtidos são importantes para o campo da saúde pública. 
A cisticercose bovina, em termos econômicos, não é uma doença de importância em nivel de criação, pois os animais apresentam infecçōes moderadas, com ausência de sintomatologia. Os prejuízos ocortem na fase final da exploração de corte, após o abate, representado, principalmente, pela condenaçāo de carcaças cisticercóticas, determinada pela fiscalização veterinária ${ }^{19}$.

Considerando-se, porém, o quadro geral da pecuária de corte, poder-se-ia interpretar a ocorrência da cisticercose bovina como um indicador da situação sanitária deste tipo de exploração, que ainda apresenta limitaçōes no seu desenvolvimento e produção, necessitando de programas adequados no sentido de superá-las $8,9,12$.

Não há dúvida quanto à necessidade de se implantar um programa de controle em nível de Estado, porém, não se pode deixar de considerar a importância da participaçāo dos municípios na realização dos mesmos, principalmente no momento em que se enfatiza a municipalização dos serviços de saúde.

A cisticercose é um problema de saúde pública, e não pode ser desconsiderado nem pelos órgãos públicos (fiscalizadores), nem pela comunidade (consumidores).

Neste sentido, o SIF, pela sua posição como centro diagnóstico de patologias, no caso, em nivel de matadouros, é um observador privilegiado e o único detentor potencial de uma massa de informaçōes. Sua contribuição, em termos de epidemiologia descritiva, é apreciável e múltipla, mas, por não processar devidamente todos os dados disponiveis, uma parte importante dessas informaçōes permanece inexplorada.

Com base nesses argumentos, o relevante papel do epidemiologista, desempenhado pelo médico veterinário inspetor, deve ser aproveitado na íntegra, lançando-se mão, o quanto antes, dos recursos de informática, para fornecer aos resposáveis pela saúde e ao conjunto da comunidade, informaçōes à altura de suas possibilidades.

UNGAR, M.L \& GERMANO, P.M.L. [Bovine cysticercosis prevalence in the State of S. Paulo, Brazil]. Rev. Saúde públ., S. Paulo, 26: 167-72, 1992. A retrospective study of Cysticercus bovis in cattle raised and slaughtered in the State of S. Paulo, Brazil, during the year of 1986, was undertaken on the basis of official records of the Brazilian Meat Inspection Service. The analysis of its spacial distribution was carried out in accordance with the political-administrative division adopted by the State Government, composed of 11 Administrative Regions (ARs) and one Metropolitan Region (MR), subdivided into 42
Governmental Regions (GRs), totaling 572 municipalities. The prevalence rates of each political-administrative unit were statistically compared using the "Z" test of two proportions. From records relating to 896,654 head, 48,957 cases of cysticercosis were detected, equivalent to a prevalence of $5.5 \%$ in the State. The prevalence results were obtained for 385 municipalities, in 42 GRs, 11 ARs and in the MR. Statistically significant prevalence values were observed in 97 municipalities, in 14 GRs and 4 ARs.

Keywords: Cysticercosis, veterinary. Cattle diseases, epidemiology. Abattoirs, standards.

\section{Referências Bibliográficas}

\section{ABDUSSALAM, M. El problema de la} teniasis-cisticercosis. In: Reunion Interamericana sobre el control de la Fiebre Aftosa y otras Zoonosis, 7., Puerto España, 1974. Washington, DC, Organización Panamericana de la Salud, 1975. p. 117-129. (OPAS - Publicación Científica, 295).

2. BARRA, A.J. \& FERREIRA, C.E. Demonstrativo de algumas doenças de bovinos transmissiveis ao homem. Hig. alim., 2:191-2, 1983.

3. BARRA, A.J. \& FERREIRA, C.E Doenças de bovinos constatadas a nível de matadouro. Hig. alim., 3: 84-6, 1984.

4. BERQUO, E.S.; SOUZA, J.M.P.; GOTLIEB, S.L.D. Bioestatistica. São Paulo, Editora Pedagógica e Universitária, 1980.

5. BOLETIM ESTATISTICO SERPA-SP. São Paulo, 1981-1986.

6. BRANT, P.C. Frequência de algumas parasitoses em carcaças e vísceras de bovinos abatidos em Belo Horizonte. Arq. UFMG, 14: 127-32, 1962.

7. CALIL, R.M. Situaçảo do complexo teníase humana cisticercose no Brasil. Comun. cient. Fac. Med. vet. Zootecn. USP, 8: 227-9, 1984.

8. CORREA, A.S. Alguns aspectos da pecuária de corte no Brasil. Campo Grande, EMBRAPA/CNPGC, 1983. (Documentos, 10).

9. CORREA, A.S. Produção e comércio de carne bovina. Campo Grande, EMBRAPA/CNPGC, 1988. (Documentos, 36).

10. COSTA, A.J.; MORI SOBRINHO, A.; AVILA, F.A.; CAVALCANTI SOBRINHO, E Distribuição geográfica da frequeencia de cisticercose bovina em algumas regiōes do Estado de São Paulo - Brasil. Cientlfica, Jaboticabal, 5: 370-9, 1977.

11. FAO. Animal health yearbook: 1977 - 1986. Rome, 1978-1987.

12. HORN, S. Programa nacional de controle de parasitoses. In: Curso sobre Parasitologia Animal, 22. Bagé, 1988. Anais. Bagé, 1988. p. 21-42.

13. INSTITUTO GEOGRAFICO E CARTOGRAFICO DE SẢo PAULO. Divisāo polftico-administrativa do Estado de São Paulo: 1986. São Paulo, 1986.

14. MACIEL, G.A. \& PAIM, G.V. Os dados nosogeográficos obtidos através do matadouro e a sua importância para a saúde pública.'Arq. Fac. Hig., 20: 241-9, 1966.

15. MOREIRA, W.S.; SANTOS, A.F.; GUARENTI, P.J. Dados epizootiológicos obtidos através do matadouro e a sua importância para a saúde pública. Rev. Centro Cienc. rurais, Santa Maria, 1: 125-32, 1971. 
16. OLIVEIRA, P.R.; RIBEIRO, S.C.A.; SILVA, P.L; REIS, D.O. Prevaléncia de cisticercose em carcacas - viscers de bovinos abatidos em Uberiindia. Arq. bres. Med. vet Zootec., 36: 443-50, 1984.

17. ORgANIZACION PANAMBRICANA DE LA SALUD. Diagnástico de la salud animal en las Américas. Washington, DC, 1983. p. 101-3. (Publicación cientúfica, 452).

18. PAWLOWSKI, Z. Taeniasis and cysticercosis. In: Jacobs, L. \& Anmbulo, P. Parasitic zoonasis. Boc Ratón, CRC Press, 1982. v.1, p. 313-48.

19. PAWLOWSKI, Z. SCHULTZ, M.G. Theniasis and cysticercosis (Taenia saginata). Adv. Parasit., 10: 269-343, 1972.

20. SChENONE, H. \& LETONA, T. Cisticercosis porcina y bovim en Latino América. Bol chil. Parasit., 29: 90-8, 1974.

21. SOULSBY, EJ.L Theniasis y cysticereosis: el problem en el viejo mundo. In: Reunion Intenmerican sobre el control de la Fiebre Aftosa y otres Zoonosis, 71, Puerto Espania, 1974. Washington, DC, Organización Panamerican de ha Salud, 1975. p. 136-42. (OPAS - Publicación Cientifica, 295).
22. UNGAR, M.L. Prevalencia da cisticercose bovina (Cysticercus bovis Goeze, 1782) do Estado de São Paulo, detectada em matadouros do referido Estado, sob o controle do Serviço de Inspecåo Federal, no ano de 1986. São Paulo, 1990. [Dissertação de Mestrado - Faculdade de Medicina Veterinária e Zootecnia da USP].

23. UNGAR, M.L; GERMANO, M.I.S.; BIGGI, G.S.; GERMANO, P.M.L. O valor dos registros de estabelecimentos de abate para a saúde pública. Comun cient. Fac. Med vet. Zootech. USP, 14: 161-5, 1990

24. VIANA, M.C. Complexo teníase-cisticercose: análise critica dos dados de ocorrencia da teníase e da cisticercose humana no Brasil, com especial referência ao Estado de Sazo Paulo. São Paulo, 1985. [Dissertagīo de Mestrado - Instituto de Ciências Biomédicas da USP].

Recebido para publicação em 7/10/1991

Reapresentado em 21/2/1992

Aprovado para publicaçäo em 27/2/1992 